\title{
Eruptive acral lentiginosis following chemotherapy for acute lymphoblastic leukaemia: a case series
}

\author{
Cathal O'Connor ${ }^{1}$, Clodagh Ryan ${ }^{2}$, and Lesley-Ann Murphy ${ }^{1}$ \\ ${ }^{1}$ South Infirmary Victoria University Hospital \\ ${ }^{2}$ Mercy University Hospital
}

April 21, 2021

\begin{abstract}
Lentigines are brown macules which represent increased proliferation of melanocytes at the dermo-epidermal junction. We report three cases of acral lentiginosis in children following chemotherapy for acute lymphoblastic leukaemia (ALL) which have persisted following cessation of chemotherapy, despite avid photoprotection.
\end{abstract}

DR CATHAL O'CONNOR ORCID 0000-0001-7084-5293

Type:

Case series

Eruptive acral lentiginosis following chemotherapy for acute lymphoblastic leukaemia: a case series

Cathal O'Connor MD ${ }^{1}$, Clodagh Ryan MD², Lesley-Ann Murphy MD ${ }^{1}$

1 Department of Dermatology, South Infirmary Victoria University Hospital, Cork

2 Department of Paediatric Haematology, Mercy University Hospital, Cork

\section{Corresponding author:}

Dr Cathal O'Connor

Department of Dermatology, South Infirmary Victoria University Hospital, Cork, Ireland

Email: drcathaloconnor@gmail.com

Telephone: 00353214926100

https://orcid.org/0000-0001-7084-5293

Twitter@oconnorcathal1

Conflict of interest: None

Financial disclosure: None

Word Count: 557 words

Figure Count: 3

Reference Count: 9 
Acknowledgements: We thank the patients and families for their permission to publish their case details.

Conflict of interest: None declared

Consent: Consent for publication was provided by the parents of the children in this case series.

\title{
Key Clinical Message
}

Children may develop acral lentiginosis following chemotherapy for acute lymphoblastic leukaemia. Subsequent development of dysplasia and melanoma in situ has been reported in these lesions, highlighting the need for clinical surveillance.

\begin{abstract}
Lentigines are brown macules which develop due to increased proliferation of melanocytes at the dermoepidermal junction. We report three cases of acral lentiginosis in children following chemotherapy for acute lymphoblastic leukaemia (ALL) which have persisted following cessation of chemotherapy, despite avid photoprotection. Generalised eruptive naevi with subsequent development of dysplastic naevi and melanoma in situ have been reported following chemotherapy, highlighting the importance of continued clinical observation.
\end{abstract}

\section{Key words}

acral lentigines, chemotherapy, leukaemia

\section{Introduction}

Lentigines are brown macules which develop due to increased proliferation of melanocytes at the dermoepidermal junction. They commonly occur in healthy people but can also be seen in genodermatoses such as Noonan syndrome with multiple lentigines. Eruptive lentigines have previously been described in the context of inflammatory dermatoses, ${ }^{1}$ phototherapy, ${ }^{2}$ and immunomodulatory therapy. ${ }^{3}$ Acral eruptive lentigines have been described following chemotherapy ${ }^{4}$ or as a paraneoplastic phenomenon. ${ }^{5}$ We report three cases of acral lentiginosis in children following chemotherapy for acute lymphoblastic leukaemia (ALL) which have persisted following cessation of chemotherapy, despite avid photoprotection. All patients remain in remission from ALL.

\section{Case series}

Patient one was a five-year-old Caucasian boy diagnosed with pre-B ALL in 2013 and treated as per the UKALL 2011 protocol regimen A. This regimen includes dexamethasone, vincristine, pegaspargase, methotrexate, mercaptopurine, doxorubicin, cyclophosphamide, and cytarabine. Following cessation of chemotherapy in 2016, multiple small brown macules were noted on the palmar and plantar surfaces of hands and feet (Figure 1). These have persisted for over four years.

Patient two was a nine-year-old Caucasian boy diagnosed with pre-B ALL in 2017 and treated as per the COG ALL 1131 protocol. This regimen includes prednisolone, vincristine, pegaspargase, methotrexate, mercaptopurine, anthracyclines, cyclophosphamide, and cytarabine. During maintenance chemotherapy in 2020, multiple small brown macules were noted on the dorsal surfaces of fingers and feet (Figure 2).

Patient three was a seven-year-old Caucasian boy diagnosed with pre-B ALL in 2018 and treated as per the UKALL 2011 protocol regimen A. During maintenance chemotherapy in 2020, multiple small brown macules were noted on the palmar and plantar surfaces of hands and feet, as well as extensive macules on the trunk (Figure 3).

\section{Discussion}

Lentigines are the most basic form of melanocytic proliferation, on a spectrum that progresses to junctional, compound, and dermal naevi. Inflammation or immunosuppression can induce melanocyte hyperplasia. Cytotoxic agents may also induce lentigines via modulation of tumour-specific lymphocytes. ${ }^{4}$ Eruptive 
lentigines have been described in the context of psoriasis, atopic dermatitis, and drug eruptions, as well as secondary to both psoralen and ultraviolet A (PUVA) and ultraviolet B (UVB) phototherapy. Topical tacrolimus and topical immunotherapy with dibutyl squaric acid have also been associated with lentigines. Drugs implicated in eruptive lentiginosis include immunomodulatory therapy such as methotrexate, azathioprine, apremilast, etanercept, adalimumab, infliximab, ustekinumab, secukimumab, and ixekizumab, and cancer chemotherapy.

Acral lentiginosis is characterised by eruptive lentigines limited to the hands and feet. The predilection for acral sites may occur due to a combination of altered immunosurveillance and elevated local trophic factors present in acral skin. ${ }^{6}$ Exposure to ultraviolet radiation may facilitate their development. Eruptive acral lentiginosis has been reported following chemotherapy for ALL in children ${ }^{7}$, and following treatment with capecitabine ${ }^{4}$ and tegafur ${ }^{8}$ (both prodrugs of 5 -fluorouracil).

There are obvious difficulties in ascertaining the culprit drug for lentiginosis when multiple cytotoxic and immunosuppressive drugs are administered concomitantly as a part of a chemotherapeutic protocol. Anecdotally, we have also noted several cases of eruptive acral lentiginosis in patients treated with high dose cytarabine for acute myeloid leukaemia (C Ryan, personal observation). Development of lentigines may depend on a complex milieu of cancer, immunosuppression, cytotoxic drug therapy, and UV exposure.

Generalised eruptive naevi with subsequent development of dysplastic naevi and melanoma in situ have been reported following chemotherapy ${ }^{9}$, highlighting the importance of continued clinical observation.

\section{References}

1 Micieli R, \& Alavi A. (2018). Eruptive lentiginosis in resolving psoriatic plaques. JAAD Case Reports, 4(9), 924-929. doi:10.1016/j.jdcr.2018.07.021

2 Friedland R, David M, Feinmesser M, Barzilai A, \& Hodak E. (2011). NB-UVB (311-312 nm)-induced lentigines in patients with mycosis fungoides: a new adverse effect of phototherapy. Journal of the European Academy of Dermatology and Venereology, 26(9), 1158-1162. doi:10.1111/j.1468-3083.2011.04189.x

3 Bovenschen HJ, Tjioe M, Vermaat H, Hoop D, Witteman BMJ., Janssens RWA., .. Kerkhof PCM. (2006). Induction of eruptive benign melanocytic naevi by immune suppressive agents, including biologicals. British Journal of Dermatology, 154(5), 880-884. doi:10.1111/j.1365-2133.2006.07189.x

4 Villalón G, Martín JM, Pinazo MI, Calduch L, Alonso V, Jordá E. (2009). Focal Acral Hyperpigmentation in a Patient Undergoing Chemotherapy with Capecitabine. American Journal of Clinical Dermatology, 10(4), 261-263. doi:10.2165/00128071-200910040-00006

5 Wolf R, Orion E, \& Davidovici B. (2008). Acral lentigines: a new paraneoplastic syndrome. International Journal of Dermatology, 47(2), 168-170. doi:10.1111/j.1365-4632.2008.03223.x

6 Woodhouse J, Maytin EV. (2005). Eruptive nevi of the palms and soles. Journal of the American Academy of Dermatology, 52(5), S96-S100. doi:10.1016/j.jaad.2004.08.009

7 Martín Hernández, JM, Donat Colomer J, Monteagudo Castro C, Fernández-Delgado Cerdá R, Alonso Usero V, Jordá Cuevas E. (2006). Nevos acrales eruptivos tras quimioterapia en niños afectados de leucemia linfoblástica aguda. Anales de Pediatría, 65(3), 260-262. doi:10.1157/13092164

8 Park YJ, Soh BW, Lee ES. Acral lentiginosis associated with tegafur/gimeracil/oteracil (TS-1). Eur $J$ Dermatol . 2017;27(2):209-210. doi:10.1684/ejd.2016.2953

9 Reutter JC, Long EM, Morrell DS, Thomas NE, Groben PA. (2007). Eruptive Post-Chemotherapy In Situ Melanomas and Dysplastic Nevi. Pediatric Dermatology, 24(2), 135-13\%. doi:10.1111/j.15251470.2007.00359.x 

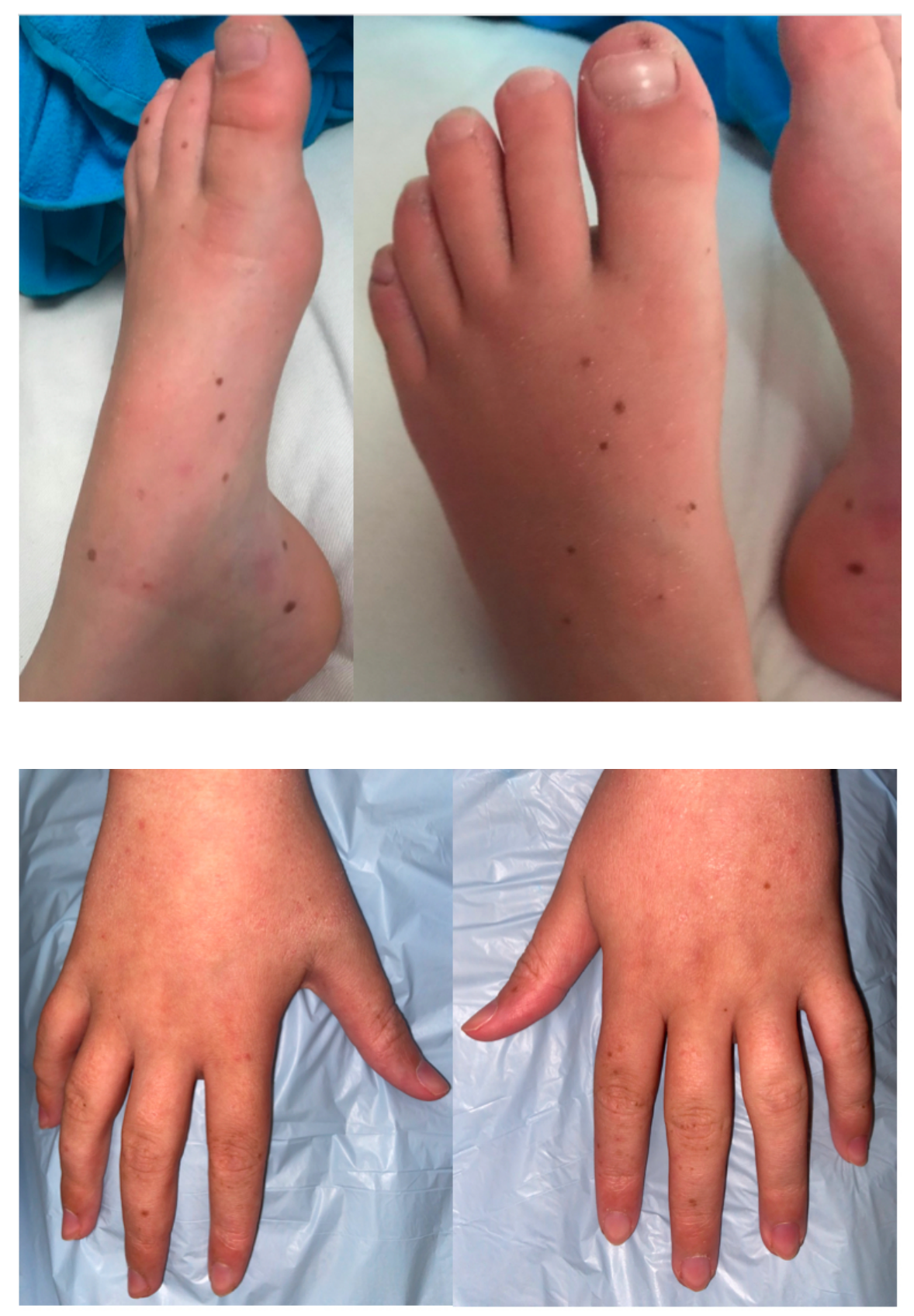

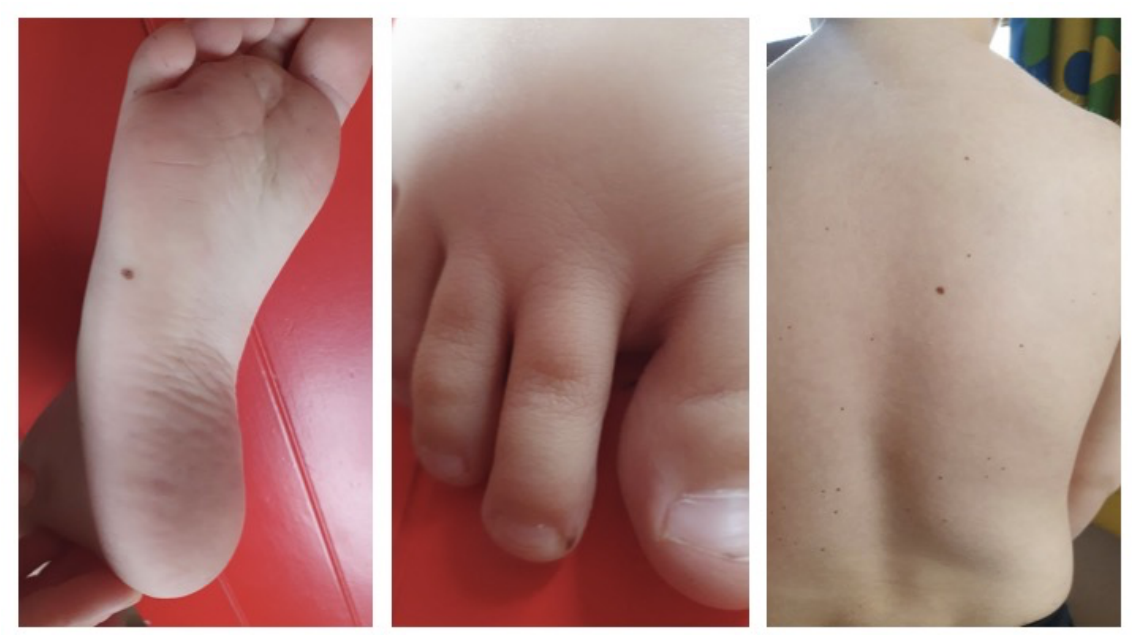